\title{
Cellular phenotype transformation occurs during thoracic aortic aneurysm development
}

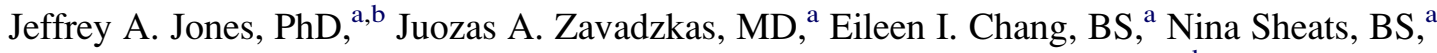 \\ Christine Koval, BS, ${ }^{a}$ Robert E. Stroud, MS, ${ }^{a}$ Francis G. Spinale, MD, PhD, ${ }^{\mathrm{a}, \mathrm{b}}$ and \\ John S. Ikonomidis, MD, $\mathrm{PhD}^{\mathrm{a}}$
}

Objective: Thoracic aortic aneurysms result from dysregulated remodeling of the vascular extracellular matrix, which may occur as a result of altered resident cellular function. The present study tested the hypothesis that aortic fibroblasts undergo a stable change in cellular phenotype during thoracic aortic aneurysm formation.

\begin{abstract}
Methods: Primary murine aortic fibroblasts were isolated from normal and thoracic aortic aneurysm-induced aortas (4 weeks post induction with $0.5 \mathrm{~mol} / \mathrm{L} \mathrm{CaCl}{ }_{2} 15$ minutes) by the outgrowth method. Normal and thoracic aortic aneurysm cultures were examined using a focused polymerase chain reaction array to determine fibroblast-specific changes in gene expression in the absence and presence of biological stimulation (endothelin-1, phorbol-12-myristate-13-acetate, angiotensin-II). The relative expression of 38 genes, normalized to 4 housekeeping genes, was determined, and genes displaying a minimum 2-fold increase/decrease or genes with significantly different normalized cycle threshold values were considered to have altered expression.
\end{abstract}

Results: At steady state, thoracic aortic aneurysm fibroblasts revealed elevated expression of several matrix metalloproteinases (Mmp2, Mmp11, Mmp14), collagen genes/elastin (Collal, Colla2, Col3al, Eln), and other matrix proteins, as well as decreased expression of $M m p 3$, Timp3, and $L t b p 1$. Moreover, gene expression profiles in thoracic aortic aneurysm fibroblasts were different than normal fibroblasts after equivalent biological stimuli.

Conclusions: This study demonstrated for the first time that isolated primary aortic fibroblasts from thoracic aortic aneurysm-induced mice possess a unique and stable gene expression profile, and when challenged with biological stimuli, induce a transcriptional response that is different from normal aortic fibroblasts. Together, these data suggest that aortic fibroblasts undergo a stable phenotypic change during thoracic aortic aneurysm development, which may drive the enhancement of extracellular matrix proteolysis in thoracic aortic aneurysm progression. (J Thorac Cardiovasc Surg 2010;140:653-9)

Thoracic aortic aneurysms (TAAs) develop in response to pathologic changes that alter the structure and composition of the aortic extracellular matrix (ECM). ${ }^{1,2}$ These dynamic changes produce an imbalance between matrix degradation and deposition resulting in compromised structural integrity and a propensity to dilate, dissect, or rupture. ${ }^{2,3}$ Although the inciting stimuli remain undefined, it has become clear that TAA development is multifactorial and involves both

From the Division of Cardiothoracic Surgery, ${ }^{a}$ Department of Surgery, Medical University of South Carolina, and the Ralph H. Johnson Veterans Affairs Medical Center, ${ }^{\mathrm{b}}$ Charleston, SC.

This work was supported by the National Institutes of Health/National Health, Lung, and Blood Institute R01 HL075488 (to J.S.I.) and R01 HL81692 (to F.G.S.), and by the Department of Veterans Affairs through a Career Development Award to J.A.J. (BLRD-CDA-2) and a Merit Award to F.G.S.

Disclosures: None.

Received for publication Aug 19, 2009; revisions received Nov 25, 2009; accepted for publication Dec 14, 2009; available ahead of print March 10, 2010.

Address for reprints: Jeffrey A. Jones, PhD, Assistant Professor/Research Health Scientist, Medical University of South Carolina, Ralph H. Johnson VA Medical Center, Cardiothoracic Surgery Research, Strom Thurmond Research Building, 114 Doughty Street, Suite 625, PO Box 250778, Charleston, SC 29425 (E-mail: jonesja@musc.edu).

0022-5223/\$0.00

Published by Elsevier Inc. on behalf of The American Association for Thoracic Surgery

doi:10.1016/j.jtcvs.2009.12.033 cellular and molecular mechanisms. Current data from clinical TAA specimens and experimental animal models have implicated the matrix metalloproteinases (MMPs) as key mediators of aneurysm formation. Although many studies have suggested that inflammatory cells are the major source of MMPs in aneurysm development, ${ }^{4,5}$ changes in MMP abundance can also arise as a result of altered production by the endogenous cellular constituents. For example, LeMaire and colleagues ${ }^{5}$ implicated a role for endogenous cells in mediating elevated MMP expression in ascending aortic aneurysm specimens from patients with bicuspid aortic valves lacking inflammatory infiltrate. Furthermore, work from this laboratory localized MMP-9 promoter activation to fibroblasts/fibroblast-derived cells within the developing murine TAA. ${ }^{6}$ With many studies reporting a loss of medial smooth muscle cells (SMCs) during TAA development, ${ }^{7,8}$ it is likely that the changes in endogenous cellular constituents play a significant role in mediating TAA formation and progression. In a recent report from this laboratory using a murine model of TAA, aortic dilatation occurred concomitantly with a loss of medial SMCs and the emergence of a subset of fibroblast-derived myofibroblasts. ${ }^{9}$

Accordingly, the present study examined the hypothesis that aortic fibroblasts undergo a phenotypic transformation that 


$$
\begin{aligned}
& \text { Abbreviations and Acronyms } \\
& \begin{array}{ll}
\text { Ang } & =\text { angiotensin } \\
\text { Ct } & =\text { cycle threshold } \\
\text { ECM } & =\text { extracellular matrix } \\
\text { ET } & =\text { endothelin } \\
\text { MMP } & =\text { matrix metalloproteinase } \\
\text { PCR } & =\text { polymerase chain reaction } \\
\text { PMA } & =\text { phorbol-12-myristate-13-acetate } \\
\text { QPCR } & =\text { quantitative polymerase chain reaction } \\
\text { SMC } & =\text { smooth muscle cell } \\
\text { TAA } & =\text { thoracic aortic aneurysm } \\
\text { TGF } & =\text { transforming growth factor }
\end{array}
\end{aligned}
$$

results in enhanced degradative capacity and the ability to compensate for the loss of SMCs in the developing aneurysm. The hypothesis was tested through 2 primary objectives. The first objective examined gene expression differences in primary aortic fibroblasts isolated from either normal mice or mice 4 weeks after TAA induction surgery. The second objective then determined whether the isolated fibroblasts responded in similar fashion when equivalently challenged with relevant biological stimuli. To accomplish these objectives, a custom quantitative real-time polymerase chain reaction array was constructed and used to simultaneously determine the relative expression of multiple determinants of ECM degradation and deposition in normal and TAA fibroblasts.

\section{MATERIALS AND METHODS \\ Experimental Design}

The present study examined gene expression in primary murine aortic fibroblasts isolated from normal and TAA-induced mice. Four C57BL/6 J mice (8-12 weeks old, equal number of male and female mice) underwent TAA induction surgery with a terminal time-point of 4 weeks post-TAA induction. At terminal surgery, the descending thoracic aorta was excised under sterile conditions and processed for fibroblast outgrowth according to established procedures. ${ }^{10}$ At the same time, aortas were harvested from 4 unoperated C57BL/6 J age- and gender-matched control mice and processed identically for fibroblast outgrowth. This animal protocol was approved by the Medical University of South Carolina Institutional Care and Use Committee, and all mice were treated and cared for in accordance with the National Institutes of Health Guide for the Care and Use of Laboratory Animals (Publication No. 85-23, revised 1996).

\section{Operative Procedure}

Murine TAAs were induced as previously described. ${ }^{11}$ Briefly, after anesthetic induction, mice were intubated and a surgical plane of anesthesia was maintained using a $2 \%$ isoflurane/oxygen mixture. The descending thoracic aorta was exposed through a left thoracotomy. A sponge soaked in $0.5 \mathrm{~mol} / \mathrm{L}$ calcium chloride was then placed in direct contact with the periadventitial surface for 15 minutes. The chest was irrigated and closed in layers, and the mice were allowed to recover.

Terminal surgical procedures and fibroblast isolation. Mice were transferred to a laminar flow biosafety cabinet and euthanized under deep anesthesia by exsanguination induced by right atriotomy. The animals were then systemically perfused with sterile saline until the perfusate was clear and the liver was blanched. The descending thoracic aorta was excised, rinsed in sterile saline, and cut longitudinally. The endothelial cell layer was removed by gently rubbing the luminal surface with a sterile swab. The aorta was then cut into approximately ten $1 \times 2 \mathrm{~mm}$ pieces and carefully placed onto the surface of a dry tissue culture flask (T-75, Cat\#13680-65; BD Falcon, Fisher Scientific, Pittsburgh, PA). The tissue was allowed to adhere in the absence of medium for 5 to 10 minutes, and then $10 \mathrm{~mL}$ of fibroblast growth medium (Fibroblast Growth Medium, Promocell Cat\#C39315, Heidelberg, Germany), containing Fibroblast Growth Supplement (Promocell Cat\#C23010; consisting of $1.0 \mathrm{ng} / \mathrm{mL}$ basic fibroblast growth factor, and $0.5 \mathrm{mg} / \mathrm{mL}$ insulin at final concentration) and $20 \%$ heat-inactivated fetal-calf serum (Invitrogen, Cat\# 10082-147, Carlsbad, CA), was carefully added to the flask. The flask was placed in a humidified $5 \% \mathrm{CO}_{2}$ incubator, and the fibroblasts were allowed to grow out of the individual tissue chunks. Evidence of fibroblast outgrowth was typically observed within 7 days of plating. Of the 8 cell lines initiated, 4 normal aortic fibroblast cell lines and 3 TAA cell lines reached confluence within $22 \pm 1$ days after plating. Fibroblasts were identified as spindle-shaped cells during log phase growth, and their identity was confirmed by staining log-phase cells with phalloidin to observe cellular architecture and with cell-type specific markers to verify purity (DDR2, prolyl-4-hydroxylase, and heavy chain myosin). The established cell lines were maintained in culture and split into new flasks when the cell density reached approximately $90 \%$ confluence. Fibroblasts in passages 3 to 6 were used for experimental studies.

Cell stimulation. For steady-state analysis, normal and TAA fibroblasts were grown to approximately $80 \%$ confluence and placed in serum-reduced medium (fibroblast growth medium + fibroblast growth supplement) containing $0.1 \%$ bovine serum albumin for 24 hours before harvest. The cells were scraped into cold $\left(4^{\circ} \mathrm{C}\right)$ phosphate-buffered saline and collected by centrifugation $(3000 \mathrm{~g})$. The cell pellet was resuspended in $300 \mathrm{~mL}$ of RNAprotect Cell Reagent (Cat\#76526, QIAGEN, Inc, Valencia, CA) and stored at $4^{\circ} \mathrm{C}$ for 24 hours.

For the cellular stimulation studies, normal and TAA fibroblasts were grown to approximately $70 \%$ confluence and then placed in serum-reduced medium containing $0.1 \%$ bovine serum albumin (as detailed above) for 24 hours. The following day, the serum-reduced medium was aspirated and replaced with $5 \mathrm{~mL}$ of the same medium containing $1 \mathrm{nM}$ endothelin (ET)-1 (Cat\# E-7764; Sigma Chemical Co, St Louis, MO), $100 \mathrm{nM}$ angiotensin (Ang)-II (Cat\# A-9525; Sigma Chemical Co), or $100 \mathrm{nM}$ phorbol12-myristate-13-acetate (PMA; Cat\# P-8139; Sigma Chemical Co). The cells were then allowed to grow for an additional 24 hours. At the end of the stimulation period, the cells were processed as detailed above.

Gene expression analysis. Total RNA was isolated using the QIAGEN RNeasy Plus Mini Kit (Cat\#74134, QIAGEN, Inc). RNA quality and quantity were analyzed with the Experion Automated Electrophoresis System (Bio-Rad Laboratories, Hercules, CA) using an Experion RNA StdSens Analysis Kit (Cat\#700-7103, Bio-Rad Laboratories). One milligram of high-quality RNA from each cell line was reverse-transcribed to generate cDNA using an RT ${ }^{2}$ First Strand Kit (Cat\#C-03, SABiosciences, Frederick, $\mathrm{MD}$ ), and the cDNA was immediately assayed for gene expression by quantitative PCR (QPCR).

To easily assess the expression of numerous genes from the established primary cell lines, a custom $\mathrm{RT}^{2}$ Profiler PCR Array (Custom Services; SABiosciences) was designed to test 42 different genes, including 4 housekeeping control genes, in a 96-well plate format. The generated cDNA was diluted into $\mathrm{RT}^{2}$ QPCR Master Mix (Cat\#PA-011, containing Hot-Start Taq polymerase and a SYBR Green/Fluorescein mix specific for Bio-Rad QPCR systems; SABiosciences), according to the manufacturer's instructions, and applied to a 96-well PCR Array plate. QPCR was performed using a MyiQ Single-Color Real-Time PCR Detection System (Bio-Rad) with the following cycling parameters: initial denaturation for 10 minutes at $95^{\circ} \mathrm{C}$ was followed by 40 cycles of 15 seconds at $95^{\circ} \mathrm{C}, 40$ seconds at $55^{\circ} \mathrm{C}$, and 30 seconds at $72^{\circ} \mathrm{C}$. A melt curve was established immediately after 
TABLE 1. List of genes assayed in normal and thoracic aortic aneurysm fibroblasts by quantitative polymerase chain reaction

\begin{tabular}{|c|c|c|c|c|c|}
\hline & Target & Gene symbol & UniGene & RefSeq & Description \\
\hline \multirow{14}{*}{ MMPs/TIMPs } & 1 & Mmp2 & Mm.29564 & NM_008610 & Matrix metallopeptidase 2 \\
\hline & 2 & Mmp3 & Mm.4993 & NM_010809 & Matrix metallopeptidase 3 \\
\hline & 3 & Mmp7 & Mm.4825 & NM_010810 & Matrix metallopeptidase 7 \\
\hline & 4 & Mmp8 & Mm.16415 & NM_008611 & Matrix metallopeptidase 8 \\
\hline & 5 & Mmp9 & Mm.4406 & NM_013599 & Matrix metallopeptidase 9 \\
\hline & 6 & Mmp11 & $\mathrm{Mm} .4561$ & NM_008606 & Matrix metallopeptidase 11 \\
\hline & 7 & Mmp12 & Mm.2055 & NM_008605 & Matrix metallopeptidase 12 \\
\hline & 8 & Mmp13 & Mm.5022 & NM_008607 & Matrix metallopeptidase 13 \\
\hline & 9 & Mmp14 & Mm.280175 & NM_008608 & Matrix metallopeptidase 14 (MT1-MMP) \\
\hline & 10 & Mmp15 & Mm.217116 & NM_008609 & Matrix metallopeptidase 15 (MT2-MMP) \\
\hline & 11 & Timpl & Mm.8245 & NM_011593 & Tissue inhibitor of metalloproteinase 1 \\
\hline & 12 & Timp2 & Mm.206505 & NM_011594 & Tissue inhibitor of metalloproteinase 2 \\
\hline & 13 & Timp3 & Mm.4871 & NM_011595 & Tissue inhibitor of metalloproteinase 3 \\
\hline & 14 & Timp4 & Mm.255607 & NM_080639 & Tissue inhibitor of metalloproteinase 4 \\
\hline \multirow[t]{17}{*}{ ECM proteins } & 15 & Collal & Mm.277735 & NM_007742 & Procollagen, type I, alpha 1 \\
\hline & 16 & Colla2 & Mm.277792 & NM_007743 & Procollagen, type I, alpha 2 \\
\hline & 17 & Col3al & Mm.249555 & NM_009930 & Procollagen, type III, alpha 1 \\
\hline & 18 & Col4al & Mm.738 & NM_009931 & Procollagen, type IV, alpha 1 \\
\hline & 19 & Col6al & Mm.2509 & NM_009933 & Procollagen, type VI, alpha 1 \\
\hline & 20 & Eln & Mm.275320 & NM_007925 & Elastin \\
\hline & 21 & Ltbpl & Mm.269747 & NM_019919 & Latent transforming growth factor-beta binding protein 1 \\
\hline & 22 & Ltbp 2 & Mm.3900 & NM_013589 & Latent transforming growth factor-beta binding protein 2 \\
\hline & 23 & Fbnl & Mm.271644 & NM_007993 & Fibrillin 1 \\
\hline & 24 & Lamb1-1 & Mm.172674 & NM_008482 & Laminin $B 1$ subunit 1 \\
\hline & 25 & Lamb2 & Mm.425599 & NM_008483 & Laminin, beta- 2 \\
\hline & 26 & Lamb3 & Mm.435441 & NM_008484 & Laminin, beta-3 \\
\hline & 27 & Fnl & Mm.193099 & NM_010233 & Fibronectin 1 \\
\hline & 28 & Sppl & Mm.288474 & NM_009263 & Secreted phosphoprotein 1 (osteopontin) \\
\hline & 29 & Thbsl & Mm.4159 & NM_011580 & Thrombospondin 1 \\
\hline & 30 & Sparc & Mm.291442 & NM_009242 & Secreted acidic cysteine rich glycoprotein (SPARC) \\
\hline & 31 & Ager & Mm.3383 & NM_007425 & Advanced glycosylation end-product receptor (RAGE) \\
\hline \multirow[t]{7}{*}{ Transcription factors } & 32 & Spl & Mm.4618 & NM_013672 & Transacting transcription factor $1(\mathrm{Sp} 1)$ \\
\hline & 33 & Sp3 & Mm.124328 & NM_011450 & Transacting transcription factor $3(\mathrm{Sp} 3)$ \\
\hline & 34 & Jun & Mm.275071 & NM_010591 & Jun oncogene (Jun) \\
\hline & 35 & Junb & Mm.1167 & NM_008416 & Jun-B oncogene (Jun b) \\
\hline & 36 & Fos & Mm.246513 & NM_010234 & FBJ osteosarcoma oncogene (Fos) \\
\hline & 37 & Fosb & Mm.248335 & NM_008036 & FBJ osteosarcoma oncogene B (Fos b) \\
\hline & 38 & Etv4 & Mm.5025 & NM_008815 & Ets variant gene 4 (E1A enhancer binding protein) (PEA-3) \\
\hline \multirow[t]{4}{*}{ House-keeping genes } & 39 & Gusb & Mm.3317 & NM_010368 & Glucuronidase, beta \\
\hline & 40 & Hprt1 & Mm.299381 & NM_013556 & Hypoxanthine guanine phosphoribosyl transferase 1 \\
\hline & 41 & Hspbl & Mm.13849 & NM_013560 & Heat shock protein 1 (Hsp27) \\
\hline & 42 & Gapdh & Mm.343110 & NM_008084 & Glyceraldehyde-3-phosphate dehydrogenase \\
\hline
\end{tabular}

MMP/TIMP, Matrix metalloproteinase/tissue inhibitor of metalloproteinase; $E C M$, extracellular matrix. Data compiled using Rebhan and colleagues' study. ${ }^{27}$

the conclusion of the cycling program to allow for confirmation of a single QPCR product for each gene-specific primer set $\left(1\right.$ minute at $95^{\circ} \mathrm{C}, 2 \mathrm{~min}-$ utes at $65^{\circ} \mathrm{C}$, followed by 60 cycles of 15 seconds at $65^{\circ} \mathrm{C}$ with an increase of $0.5^{\circ} \mathrm{C}$ per cycle). Negative controls were run on each plate to verify the absence of genomic DNA contamination (no reverse transcription control) and the absence of overall DNA contamination in the PCR system and working environment (no template control).

Data analysis. Cycle threshold $(\mathrm{Ct})$ values were recorded and fold change in steady-state gene expression between TAA fibroblasts and normal fibroblasts was calculated using the $\Delta \Delta \mathrm{Ct}$ method (see Table 1 for a complete listing of genes examined, including housekeeping genes). Fold expression values greater than 2.0 or less than 0.5 were considered to have a significant change in gene expression. In addition, any gene that displayed a significant difference in mean $\Delta \mathrm{Ct}$ value (TAA vs normal by 2-sided, 2-tailed $t$ test; $P<.05$ ) was likewise considered to have a significant change in expression. Ct values of 35.0 or greater were considered non-cycling and were removed from analysis. Of the genes tested, primers for Mmp8, Mmp12, Mmp7, and Timp4 consistently produced multiple peaks on melt-curve analysis indicating that more than 1 product was being amplified in those wells. Accordingly, these genes were also removed from analysis. In addition, Mmp9, Timp1, and Lamb3 did not consistently cycle because of low target concentration or poor primer design. To measure Mmp9 and Timp1 gene expression, TaqMan primer/probe sets were used with same QPCR conditions indicated above (Mmp9, Cat\# 
TABLE 2. Relative fold gene expression in thoracic aortic aneurysm fibroblasts compared with normal fibroblasts at steady-state and after stimulation with various growth factors at the indicated concentrations

\begin{tabular}{|c|c|c|c|c|c|}
\hline & \multirow[b]{2}{*}{ Gene symbol } & \multicolumn{4}{|c|}{$\begin{array}{c}\text { Fold expression } \\
\text { (TAA fibroblasts vs normal fibroblasts) }\end{array}$} \\
\hline & & Steady-state & ET-1 (1 nM) & PMA (100 nM) & AngII (100 nM) \\
\hline \multirow[t]{10}{*}{ MMPs/TIMPs } & Mmp2 & $6.54(P=.0055)$ & $4.46(P=.0239)$ & $3.65(P=.0472)$ & $5.94(P=.0171)$ \\
\hline & Mmp3 & $0.41(P=.3622)$ & $0.26(P=.2470)$ & $0.06(P=.0137)$ & $0.22(P=.2382)$ \\
\hline & Mmp9 & $0.09(P=.0488)$ & $0.04(P=.0012)$ & $0.21(P=.1109)$ & $0.09(P=.1828)$ \\
\hline & Mmpl1 & $9.78(P=.0027)$ & $2.78(P=.2959)$ & $2.03(P=.1184)$ & $7.26(P=.0278)$ \\
\hline & Mmpl3 & $1.11(P=.8461)$ & $0.97(P=.9514)$ & $0.11(P=.0280)$ & $0.42(P=.7376)$ \\
\hline & Mmp14 & $2.01(P=.1014)$ & $2.13(P=.0008)$ & $1.78(P=.1450)$ & $1.87(P=.1579)$ \\
\hline & Mmp15 & $1.17(P=.8590)$ & $0.66(P=.3911)$ & Dec & $0.23(P=.0902)$ \\
\hline & Timpl & $1.41(P=.2415)$ & $0.99(P=.9766)$ & $1.53(P=.1271)$ & $1.39(P=.4637)$ \\
\hline & Timp2 & $1.11(P=.5787)$ & $0.95(P=.8977)$ & $0.95(P=.8377)$ & $1.27(P=.3828)$ \\
\hline & Timp3 & $0.33(P=.1490)$ & $0.49(P=.3012)$ & $0.43(P=.0799)$ & $0.30(P=.0896)$ \\
\hline \multirow[t]{9}{*}{ Collagen/elastic architecture } & Collal & $2.52(P=.0583)$ & $1.83(P=.0793)$ & $2.99(P=.0015)$ & $1.84(P=.2291)$ \\
\hline & Colla2 & $3.45(P=.0082)$ & $2.90(P=.0007)$ & $3.61(P=.0047)$ & $3.46(P=.0049)$ \\
\hline & Col3al & $4.83(P=.0083)$ & $3.51(P=.0102)$ & $4.52(P=.0120)$ & $4.05(P=.0109)$ \\
\hline & Col4al & $2.82(P=.0039)$ & $2.36(P=.0284)$ & $2.22(P=.3715)$ & $1.94(P=.4345)$ \\
\hline & Col6al & $1.67(P=.2485)$ & $1.47(P=.4708)$ & $1.52(P=.5061)$ & $1.41(P=.4827)$ \\
\hline & Eln & $2.30(P=.2558)$ & $2.32(P=.2918)$ & $0.79(P=.7256)$ & $2.24(P=.4751)$ \\
\hline & Ltbp-1 & $0.09(P=.0564)$ & $0.21(P=.0323)$ & $0.04(P=.0033)$ & $0.19(P=.0955)$ \\
\hline & $L t b p-2$ & $1.71(P=.2016)$ & $1.39(P=.4174)$ & $1.44(P=.3357)$ & $1.63(P=.3735)$ \\
\hline & Fbnl & $1.83(P=.2042)$ & $2.13(P=.1269)$ & $2.33(P=.1862)$ & $2.55(P=.1643)$ \\
\hline \multirow[t]{7}{*}{ Other ECM } & Lamb1-1 & $1.66(P=.1437)$ & $1.61(P=.3753)$ & $0.99(P=.9830)$ & $2.02(P=.1812)$ \\
\hline & Lamb2 & $21.96(P=.0378)$ & $1.36(P=.7058)$ & $0.05(P=.0194)$ & $0.56(P=.3979)$ \\
\hline & Fnl & $2.23(P=.1582)$ & $1.96(P=.1730)$ & $1.56(P=.3862)$ & $1.83(P=.4078)$ \\
\hline & Sppl & $2.43(P=.2790)$ & $3.15(P=.3129)$ & $0.17(P=.6975)$ & $1.15(P=.9537)$ \\
\hline & Thbsl & $0.88(P=.5526)$ & $0.59(P=.0240)$ & $1.39(P=.2732)$ & $0.70(P=.3291)$ \\
\hline & Sparc & $2.10(P=.1208)$ & $2.25(P=.0624)$ & $1.40(P=.5110)$ & $0.90(P=.8262)$ \\
\hline & Ager & $0.72(P=.4922)$ & $0.69(P=.5648)$ & $0.73(P=.5835)$ & $0.90(P=.8769)$ \\
\hline \multirow[t]{7}{*}{ Transcription factors } & Spl & $1.68(P=.1245)$ & $1.21(P=.5112)$ & $1.22(P=.5883)$ & $0.97(P=.8670)$ \\
\hline & Sp3 & $0.98(P=.9197)$ & $1.13(P=.7552)$ & $1.03(P=.9300)$ & $0.94(P=.8385)$ \\
\hline & Jun & $1.49(P=.6169)$ & $3.71(P=.0106)$ & $2.63(P=.0460)$ & $2.28(P=.0174)$ \\
\hline & Junb & $0.82(P=.7838)$ & $1.28(P=.7831)$ & $1.46(P=.4151)$ & ND \\
\hline & Fos & $2.14(P=.7734)$ & $1.71(P=.4049)$ & $0.46(P=.4656)$ & $0.97(P=.9632)$ \\
\hline & Fosb & $2.41(P=.3959)$ & $3.40(P=.2602)$ & $5.21(P=.1182)$ & $1.67(P=.6711)$ \\
\hline & Etv4 & $0.91(P=.7819)$ & $1.14(P=.7663)$ & $0.44(P=.2232)$ & $0.82(P=.6757)$ \\
\hline
\end{tabular}

$M M P / T I M P$, Matrix metalloproteinase/tissue inhibitor of metalloproteinase; TAA, thoracic aortic aneurysm; $E C M$, extracellular matrix; $E T$, endothelin; $P M A$, phorbol-12-myristate-13-acetate; AngII, angiotensin-II; Dec, decreased below detection with stimulation; $N D$, not detectable with stimulation.

Mm00442991_m1; Timp1, Cat\# Mm00441818_m1; Applied Biosystems, Foster City, CA). There were 2 incidents of stimulus-induced increase in Ct values (PMA, Mmp15; and AngII, Junb). In PMA-treated cells, each stimulated control cell line yielded a measurable Ct value for Mmp15, whereas each stimulated TAA cell line produced $\mathrm{Ct}$ values of greater than 35.0. In AngII-treated cells, Junb expression was suppressed in both normal and TAA fibroblasts after stimulation.

Gene expression in each stimulated cell line was determined in a similar manner. To more easily represent the expression differences between normal and TAA fibroblasts, genes were clustered into similar gene families, and total gene expression was depicted as an area profile on a radar plot. Each axis displays the sum of the relative fold change in gene expression for a given gene cluster in the TAA fibroblasts compared with the control fibroblasts. The genes included in each cluster were 1) MMP/TIMP genes (Mmp2, Mmp3, Mmp9, Mmp11, Mmp13, Mmp14, Mmp15, Timp1, Timp2, Timp3); 2) collagen/elastic architecture genes (Colla1, Colla2, Col3al, Col4al, Col6al, Eln, Ltbp1, Ltbp2, Fbn1); and 3) other ECM genes (Lamb1-1, Lamb2, Lamb3, Fn1, Spp1, Thbs1, Sparc, Ager).

\section{RESULTS}

\section{Relative Gene Expression Analysis at Steady-State}

Primary aortic fibroblasts from normal and TAA-induced mice were analyzed to determine the relative expression of 38 genes and 4 housekeeping genes (Table 1) under steady-state culture conditions using a single custom QPCR array plate per cell line. The TAA mice displayed a relative increase in Mmp2, Mmpl1, and Mmpl4 expression, and a relative decrease of Mmp3, Mmp9, and Timp3 expression (Table 2, Steady-State). Furthermore, the steadystate expression of several collagen genes and elastin was also elevated (Collal, Colla2, Colla3, Col4al, and Eln), whereas the expression of $L t b p-1$ was decreased (Table 2). In addition, several other ECM proteins displayed elevated expression (Lamb2, Fnl, Sppl, and Sparc) (Table 2). Last, 


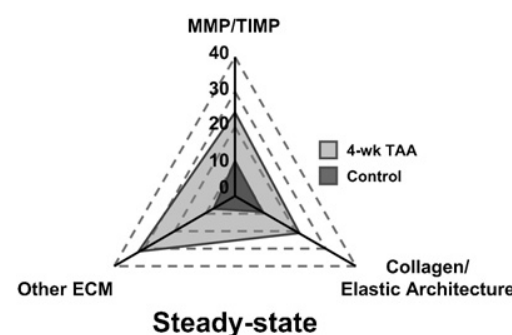

Steady-state

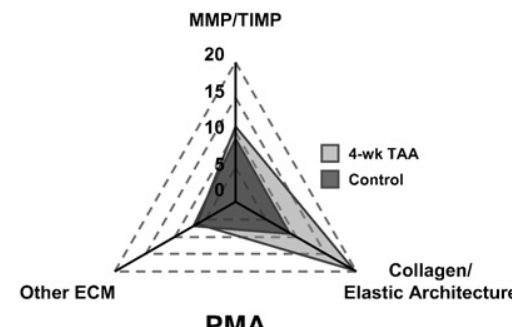

PMA
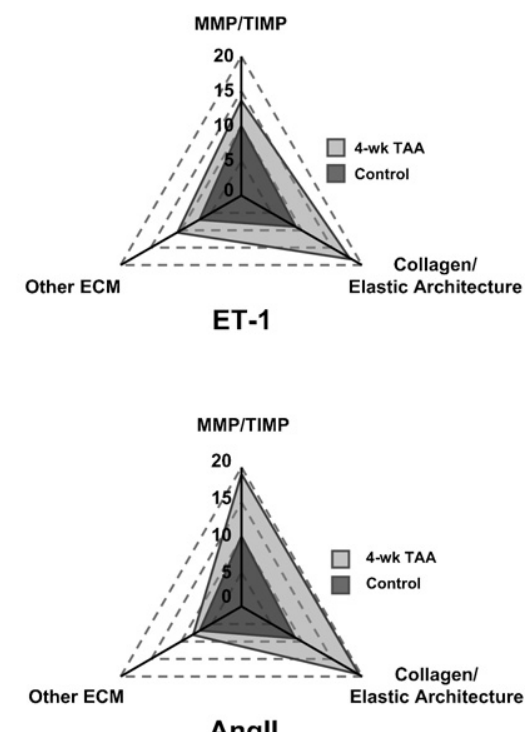

Angll

FIGURE 1. Analysis of gene expression after cellular stimulation. Relative gene expression in normal and TAA fibroblast cell lines was analyzed in the absence (steady-state) or presence of biological stimuli. Cells were treated with $1 \mathrm{nM}$ ET-1, $100 \mathrm{nM}$ AngII, or $100 \mathrm{nM}$ PMA for 24 hours and then analyzed by QPCR. Relative fold expression results were clustered in functional groups (MMP/TIMP, collagen/elastin, other ECM), and total gene expression was depicted as an area profile on a radar plot. Each axis displays the sum of the relative fold change in gene expression for a given gene cluster in the TAA fibroblasts compared with the control fibroblasts. MMP/TIMP, Matrix metalloproteinase/tissue inhibitor of metalloproteinase; TAA, thoracic aortic aneurysm; $E C M$, extracellular matrix; ET, endothelin; PMA, phorbol-12-myristate-13-acetate; AngII, angiotensin-II.

the relative expression of several transcription factors known to be involved in regulating matrix turnover was assessed. The TAA fibroblasts demonstrated increased steady-state expression of Fos and Fosb (Table 2).

\section{Relative Gene Expression Analysis After Stimulation With Endothelin-1, Phorbol-12-Myristate-13- Acetate, and Angiotensin-II}

To determine whether normal and TAA fibroblasts respond in similar fashion to equivalent biological stimuli (ET-1, PMA, AngII), both sets of fibroblasts were stimulated for 24 hours, and the relative changes in gene expression were examined. In almost all cases, the TAA fibroblasts responded more robustly than the normal fibroblasts to biological stimulus (Table 2, ET-1, PMA, AngII). To more easily demonstrate the relative changes in gene expression, the results in Table 2 were further analyzed by cluster analysis in the following functional groups: MMP/TIMP, collagen/elastic architecture, and other ECM. Figure 1 reveals the relative fold expression results for normal and TAA fibroblasts showing that the gene expression profiles for the TAA fibroblasts were different from normal fibroblasts at steady-state and after exposure to equivalent biological stimuli.

\section{DISCUSSION}

Previous clinical and experimental aneurysm studies have demonstrated significant changes in aortic structure and composition in the developing TAA. These changes include disruption of the medial elastic lamellae, ${ }^{12,13}$ alterations in collagen deposition, ${ }^{12-14}$ changes in cellular content characterized by the loss of SMCs, ${ }^{7,8}$ and alterations in aortic transcriptional profiles. ${ }^{6,15,16}$ In addition, previous studies from this laboratory have identified fibroblasts/fibroblast-derived cells as a potential source for enhanced MMP transcriptional activity during TAA development in mice. ${ }^{6,9}$ Taken together, this led us to hypothesize that aortic fibroblasts are an important cellular component within the aortic wall, which undergo a stable phenotypic change during TAA development, allowing them to adapt to the rapidly degrading conditions and mediate the vascular remodeling process. To test this hypothesis, primary thoracic aortic fibroblasts were isolated from normal and TAA-induced mice, and transcriptional profiles of key determinants of matrix degradation and deposition were assessed in the absence and presence of relevant biological stimuli. The unique outcomes of this study are 3-fold. First, the present study demonstrated significant transcriptional changes in TAA fibroblasts compared with normal aortic fibroblasts. These results are consistent with expectations of enhanced degradation and remodeling of the vascular ECM during TAA development that may ensue, in part, because of altered fibroblast function. Second, when isolated fibroblasts were challenged by treatment with relevant biological stimuli (ET-1, AngII, PMA), differential transcriptional responses were observed between normal and TAA fibroblasts, suggesting that intracellular signaling pathways in the TAA fibroblasts may be altered, and thus may respond differently to equivalent stimuli. Last, the observed gene expression differences between normal and TAA 
fibroblasts were evident in multiple cell lines after the several cell passages required to establish the in vitro cultures. By using an established fibroblast outgrowth procedure, ${ }^{10}$ multiple primary cell lines from both normal and TAA-induced animals were established in vitro. The highly reproducible transcriptional differences between the TAA and the normal fibroblasts are therefore not a by-product of selection during in vitro establishment or a direct result of cell passaging, but due to a stable alteration in transcriptional activity induced during the process of aneurysm development in vivo. Taken together, the results of this study support the hypothesis that aortic fibroblasts undergo a stable phenotypic transformation during TAA development, and that these changes in gene expression may alter normal fibroblast function and contribute to TAA formation and progression.

\section{Steady-State Changes Gene Expression}

To assess transcriptional differences between normal and TAA fibroblasts, QPCR was performed using a custom-designed PCR array to assess the gene expression of several critical determinants of matrix degradation and deposition, known to be involved in TAA development. Previous studies from this laboratory have examined aortic tissue from normal and TAA mice, and have demonstrated TAA-dependent changes in gene transcription and protein abundance of MMPs and transforming growth factor (TGF) $-\beta$ pathway components. ${ }^{6,15}$ Accordingly, it was hypothesized that if the aortic fibroblast plays a dominant role in TAA development, isolated aortic fibroblasts from TAA tissue would likewise display an altered transcriptional profile compared with normal aortic fibroblasts. Indeed, our results were consistent with that hypothesis. The TAA fibroblasts displayed a distinct pattern of gene expression reflecting enhanced expression of several MMPs (Mmp2, Mmp11, and Mmp14), matrix proteins (Colla1, Colla2, Col3a1, Col4al, Eln, Lamb2, Fn1, Spp1, and Sparc), and transcription factors (Fos and Fosb), along with the decreased expression of Mmp3, Mmp9, Timp3, and Ltbpl. The observed elevated expression of MMP genes was highly consistent with previous studies from this laboratory demonstrating a direct role for increased MMP production during TAA development in this model. ${ }^{6,17}$ Thus, the stable elevation of MMP transcription in TAA fibroblasts argues that this differentiated cell-type plays a critical role in degrading the ECM during TAA formation and progression.

Although many studies analyzing clinical TAA specimens have demonstrated elevated collagenase activity (MMP-dependent), ${ }^{1,5,18,19}$ few have examined aortic collagen content or expression directly. ${ }^{14,20}$ The present study demonstrates a robust elevation of collagen gene expression in the TAA fibroblasts. Although counterintuitive to the enhanced ECM degradation that takes place during TAA development, others have described elevated collagen expression and regional deposition in TAA tissue. For example, Iliopoulos and coworkers ${ }^{13}$ have suggested that colla- gen content, although diminished in the aortic media, is elevated in the adventitial region of the aorta, resulting in no net gain or loss of total collagen content. Similarly, Della Corte and colleagues ${ }^{12}$ have demonstrated regional differences in collagen content in the greater versus lesser curvature of the ascending aorta from aneurysmal patients with bicuspid aortic valves. Collectively, these studies suggest that the enhancement of collagen expression may coincide with TAA development and may result in the regional deposition of newly formed collagen fibers. In a recent report from this laboratory, medial and adventitial collagen content were measured over the 16-week time-course of TAA development in this model. ${ }^{9}$ No net change was observed in either the medial or adventitial compartment. Although this may suggest a lack of collagen degradation, given the present results demonstrating elevated MMP and collagen gene expression in the isolated TAA fibroblasts, it is more likely that collagen degradation and deposition are balanced in regard to the total content. This is further supported by the observation that Sparc (secreted protein acidic and rich in cysteine) expression is elevated in the TAA fibroblasts, suggesting that these cells possess a synthetic phenotype, primed to deposit newly processed collagen fibers. The organizational structure of the collagen matrix, on the other hand, may be significantly remodeled during TAA development, as has been demonstrated in the postinfarct myocardium. ${ }^{21}$ Of interest, the present study also identified decreased expression of Ltbp- 1 (latent TGF- $\beta$ binding protein-1), an ECM structural protein that serves to bind and sequester the small latent complex of TGF- $\beta$. TGF- $\beta$ is best known for its ability to induce collagen gene expression but may also play a role in exacerbating vascular remodeling during TAA development. ${ }^{22}$ Accordingly, it follows that a decrease in $L t b p-1$ expression may lead to an increase in the extracellular levels of TGF- $\beta$ and enhancement of collagen gene expression. Moreover, elevated TGF- $\beta$ signaling has also been associated with the induction of MMP expression and abundance. ${ }^{23,24}$

To further demonstrate the stability of the observed phenotypic change between isolated normal and TAA fibroblasts, the expression levels of several transcription factors implicated in regulating MMP-dependent transcription were assessed. Notably, Fos and Fosb, family members and components of the AP-1 transcription factor, were both stably induced and may play a direct role in the observed change in transcriptional profile of the TAA fibroblasts.

\section{Stimulated Changes in Gene Expression}

In an effort to further define the phenotypic change of the TAA fibroblasts, gene expression profiles were also determined after treatment with relevant biological stimuli (ET-1, ${ }^{25}$ AngII, ${ }^{26}$ PMA). When comparing relative gene expression between treated normal and TAA fibroblasts within gene family clusters, clear differences in overall transcriptional profiles were observed after treatment with each agent. 
Although it was anticipated that the MMP genes in the TAA fibroblasts would be robustly induced in response to some stimuli (eg, ET-1, PMA), the blunted response observed may be a direct result of altered downstream signaling pathways. Given that these genes were already highly expressed in the TAA fibroblast, this may further suggest that the intracellular signaling pathways have become uncoupled from receptor activation in these cells. These results further substantiate the hypothesis that the TAA fibroblasts have undergone a stable phenotypic change and may suggest that the alterations in transcriptional response are a direct result of reconfigured intracellular signaling pathways in the TAA fibroblasts.

\section{Limitations}

Because the present study examines gene expression profiles in cultured primary aortic cell lines, several limitations must be noted. First, care must be taken in extrapolating changes in gene expression with altered protein levels. Although increased or decreased gene expression often equates to coordinate changes protein translation, transcriptional and translational regulation must be considered. Second, although this murine model of TAA has been well described and recapitulates many of the hallmarks of human aneurysmal disease, some aspects such as atherosclerosis and intraluminal thrombosis, are not replicated at the time-points studied. Accordingly, care should be taken in the extrapolation of these results to human TAAs. Last, although a significant effect of gender on aortic dilatation has not previously been demonstrated in this model system, gender-dependent differences in aortic gene expression cannot be ruled out. Additional studies will be required to ascertain whether a significant interaction between gender and gene expression exist.

\section{CONCLUSIONS}

Despite limitations, the present study demonstrated for the first time that isolated primary aortic fibroblasts from TAA mice possess a stable and unique phenotype defined by altered gene expression profiles at steady-state and in response to biological stimuli. This distinctive cellular population may emerge as the major cellular mediator of vascular remodeling during TAA development and may drive the enhancement of ECM proteolysis in TAA progression.

\section{References}

1. Barbour JR, Spinale FG, Ikonomidis JS. Proteinase systems and thoracic aortic aneurysm progression. J Surg Res. 2007;139:292-307.

2. Dapunt OE, Galla JD, Sadeghi AM, Lansman SL, Mezrow CK, de Asla RA, et al. The natural history of thoracic aortic aneurysms. $J$ Thorac Cardiovasc Surg. 1994;107:1323-33.

3. Davies RR, Gallo A, Coady MA, Tellides G, Botta DM, Burke B, et al. Novel measurement of relative aortic size predicts rupture of thoracic aortic aneurysms. Ann Thorac Surg. 2006;81:169-77.

4. Freestone T, Turner RJ, Coady A, Higman DJ, Greenhalgh RM, Powell JT. Inflammation and matrix metalloproteinases in the enlarging abdominal aortic aneurysm. Arterioscler Thromb Vasc Biol. 1995;15:1145-51.
5. LeMaire SA, Wang X, Wilks JA, Carter SA, Wen S, Won T, et al. Matrix metalloproteinases in ascending aortic aneurysms: bicuspid versus trileaflet aortic valves. J Surg Res. 2005;123:40-8.

6. Jones JA, Barbour JR, Lowry AS, Bouges S, Beck C, McClister DM Jr, et al. Spatiotemporal expression and localization of matrix metalloproteinase- 9 in a murine model of thoracic aortic aneurysm. J Vasc Surg. 2006;44:1314-21.

7. Della Corte A, Quarto C, Bancone C, Castaldo C, Di Meglio F, Nurzynska D, et al. Spatiotemporal patterns of smooth muscle cell changes in ascending aortic dilatation with bicuspid and tricuspid aortic valve stenosis: focus on cell-matrix signaling. J Thorac Cardiovasc Surg. 2008;135:8-18. e1-2.

8. Ihling C, Szombathy T, Nampoothiri K, Haendeler J, Beyersdorf F, Uhl M, et al. Cystic medial degeneration of the aorta is associated with p53 accumulation, Bax upregulation, apoptotic cell death, and cell proliferation. Heart. 1999;82:286-93.

9. Jones JA, Beck C, Barbour JR, Zavadzkas JA, Mukherjee R, Spinale FG, et al. Alterations in aortic cellular constituents during thoracic aortic aneurysm development: myofibroblast-mediated vascular remodeling. Am J Pathol. 2009;175:1746-56.

10. Lindsey ML, Goshorn DK, Squires CE, Escobar GP, Hendrick JW, Mingoia JT, et al Age-dependent changes in myocardial matrix metalloproteinase/tissue inhibitor of metalloproteinase profiles and fibroblast function. Cardiovasc Res. 2005;66:410-9.

11. Ikonomidis JS, Gibson WC, Gardner J, Sweterlitsch S, Thompson RP, Mukherjee R, et al. A murine model of thoracic aortic aneurysms. J Surg Res. 2003;115:157-63.

12. Della Corte A, De Santo LS, Montagnani S, Quarto C, Romano G, Amarelli C, et al. Spatial patterns of matrix protein expression in dilated ascending aorta with aortic regurgitation: congenital bicuspid valve versus Marfan's syndrome. J Heart Valve Dis. 2006;15:20-7.

13. Iliopoulos DC, Kritharis EP, Giagini AT, Papadodima SA, Sokolis DP. Ascending thoracic aortic aneurysms are associated with compositional remodeling and vessel stiffening but not weakening in age-matched subjects. J Thorac Cardiovasc Surg. 2009;137:101-9.

14. Maleszewski JJ, Miller DV, Lu J, Dietz HC, Halushka MK. Histopathologic findings in ascending aortas from individuals with Loeys-Dietz syndrome (LDS). Am J Surg Pathol. 2009;33:194-201.

15. Jones JA, Barbour JR, Stroud RE, Bouges S, Stephens SL, Spinale FG, et al. Altered transforming growth factor-beta signaling in a murine model of thoracic aortic aneurysm. J Vasc Res. 2008;45:457-68.

16. Taketani T, Imai Y, Morota T, Maemura K, Morita H, Hayashi D, et al. Altered patterns of gene expression specific to thoracic aortic aneurysms: microarray analysis of surgically resected specimens. Int Heart J. 2005;46:265-77.

17. Barbour JR, Stroud RE, Lowry AS, Clark LL, Leone AM, Jones JA, et al. Temporal disparity in the induction of matrix metalloproteinases and tissue inhibitors of metalloproteinases after thoracic aortic aneurysm formation. J Thorac Cardiovasc Surg. 2006;132:788-95.

18. Ikonomidis JS, Jones JA, Barbour JR, Stroud RE, Clark LL, Kaplan BS, et al. Expression of matrix metalloproteinases and endogenous inhibitors within ascending aortic aneurysms of patients with Marfan syndrome. Circulation. 2006;114(1 Suppl):I365-70.

19. Ikonomidis JS, Jones JA, Barbour JR, Stroud RE, Clark LL, Kaplan BS, et al. Expression of matrix metalloproteinases and endogenous inhibitors within ascending aortic aneurysms of patients with bicuspid or tricuspid aortic valves. $J$ Thorac Cardiovasc Surg. 2007;133:1028-36.

20. de Figueiredo Borges L, Jaldin RG, Dias RR, Stolf NA, Michel JB, Gutierrez PS Collagen is reduced and disrupted in human aneurysms and dissections of ascending aorta. Hum Pathol. 2008;39:437-43.

21. Spinale FG. Myocardial matrix remodeling and the matrix metalloproteinases: influence on cardiac form and function. Physiol Rev. 2007;87:1285-342.

22. Jones JA, Spinale FG, Ikonomidis JS. Transforming growth factor-beta signaling in thoracic aortic aneurysm development: a paradox in pathogenesis. I Vasc Res. 2009;46:119-37.

23. Kim ES, Kim MS, Moon A. TGF-beta-induced upregulation of MMP-2 and MMP-9 depends on $\mathrm{p} 38 \mathrm{MAPK}$, but not ERK signaling in MCF10A human breast epithelial cells. Int J Oncol. 2004;25:1375-82.

24. Safina A, Vandette E, Bakin AV. ALK5 promotes tumor angiogenesis by upregulating matrix metalloproteinase-9 in tumor cells. Oncogene. 2007;26:2407-22.

25. Wagner A, Domanovits H, Holzer M, Kofler J, Roggla M, Mullner M, et al. Plasma endothelin in patients with acute aortic disease. Resuscitation. 2002;53:71-6.

26. Daugherty A, Rateri DL, Cassis LA. Role of the renin-angiotensin system in the development of abdominal aortic aneurysms in animals and humans. Ann NY Acad Sci. 2006;1085:82-91

27. Rebhan M, Chalifa-Caspi V, Prilusky J, Lancet D. GeneCards: integrating information about genes, proteins and diseases. Trends Genet. 1997;13:163. 